\title{
An Intact Insect Embryo for Developmental Neurotoxicity Testing of Directed Axonal Elongation
}

\author{
Gregor A. Bergmann", Sarah Frömbling\#, Nina Joseph, Karsten Bode, Gerd Bicker* and Michael Stern* \\ University of Veterinary Medicine Hannover, Institute for Physiology and Cell Biology, Hannover, Germany
}

\begin{abstract}
Developmental neurotoxicity (DNT) of chemicals poses a serious threat to human health worldwide. Current in vivo test methods for assessing DNT require the use of large numbers of laboratory animals. Most alternative testing methods monitor readily quantifiable toxicological endpoints in cell culture, whereas the formation of a functional brain requires precisely timed navigation of axons within a complex tissue environment. We address this complexity by monitoring defects in axonal navigation of pioneer axons of intact locust embryos after exposure to chemicals. Embryos develop in serum-free culture with test chemicals, followed by immunolabeling of pioneer neurons. Defects in axon elongation of pioneer axons are quantified in concentration-response curves and compared to the general viability of the embryo, as measured by a resazurin assay.

We show that selected chemical compounds interfering with calcium signaling or cytoskeletal organization, and the reference developmental neurotoxicant rotenone, can be classified as DNT positive. The pesticide rotenone inhibits pioneer neuron elongation with a lower IC50 than viability. The rho kinase inhibitor Y27632 can partially rescue outgrowth inhibition, supporting the classification of rotenone as a specific DNT positive compound. Since mechanisms of axonal guidance, such as growth cone navigation along molecular semaphorin gradients are conserved between locust and mammalian nervous systems, we will further explore the potential of this invertebrate preparation as an assay, including a prediction model, for testing the DNT potential of chemicals in humans.
\end{abstract}

\section{Introduction}

Developmental neurotoxicity (DNT) of environmental chemicals poses a serious threat to human health worldwide, and the resulting neurological deficits, in particular in children, negatively affect families and society. However, far too few chemicals have yet been tested, mainly because current in vivo test methods for assessing DNT require the use of large numbers of laboratory animals. In the last decade, there has been significant progress in the field of in vitro alternatives to animal models in DNT testing (Aschner et al., 2017; Bal-Price et al., 2018a; Coecke et al., 2007; Crofton et al., 2011; Delp et al., 2018; Fritsche et al., 2015, 2017, 2018; Lein et al., 2005; Stern et al., 2014), based on rat primary cells, human cell lines, or stem/progenitor based models.

However, alternative in vitro testing methods monitor mainly readily quantifiable toxicological endpoints, such as cell viability, proliferation, neurochemical differentiation, migration of neural precursor cells, and electrical activity in randomly formed neural networks (Frank et al., 2018). Most in vitro systems do not recapitulate many of the complex cell-cell and cell-matrix interactions or morphogen gradients in the intact organism that are necessary for normal brain formation and may be subject to significant influence by toxicants (Lein et al., 2005). To address possible adverse effects of chemicals on these complex mechanisms, non-mammalian models such as zebrafish embryos can be successfully employed (Dach et al., 2019). Alternatively, the applicability of invertebrate models such as Caenorhabditis (Avila et al., 2012), planarians (Hagstrom et al., 2019), or Drosophila (Rand, 2010 ) is recognized. In spite of the phylogenetical distance between vertebrates and invertebrates, mechanisms of neural development appear to be highly conserved (Sánchez-Soriano et al., 2007). Comparative DNT studies between a zebrafish and a \#contributed equally; * contributed equally

Received January 29, 2019; Accepted May 28, 2019; Epub May 29, 2019; @ The Authors, 2019.

ALTEX 36(4), 643-649. doi:10.14573/altex.1901292

Correspondence: Gerd Bicker, PhD, Michael Stern, PhD

University of Veterinary Medicine Hannover, Institute for Physiology and Cell Biology

Bischofsholer Damm 15/102, 30173 Hannover, Germany

(Gerd.Bicker@tiho-hannover.de; Michael.stern@tiho-hannover.de)
This is an Open Access article distributed under the terms of the Creative Commons Attribution 4.0 International license (http://creativecommons.org/licenses/by/4.0/), which permits unrestricted use, distribution and reproduction in any medium, provided the original work is appropriately cited. 
A

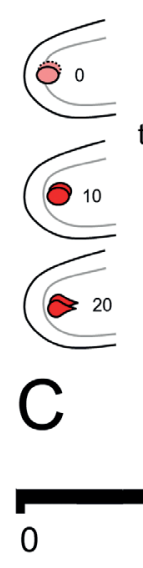

B

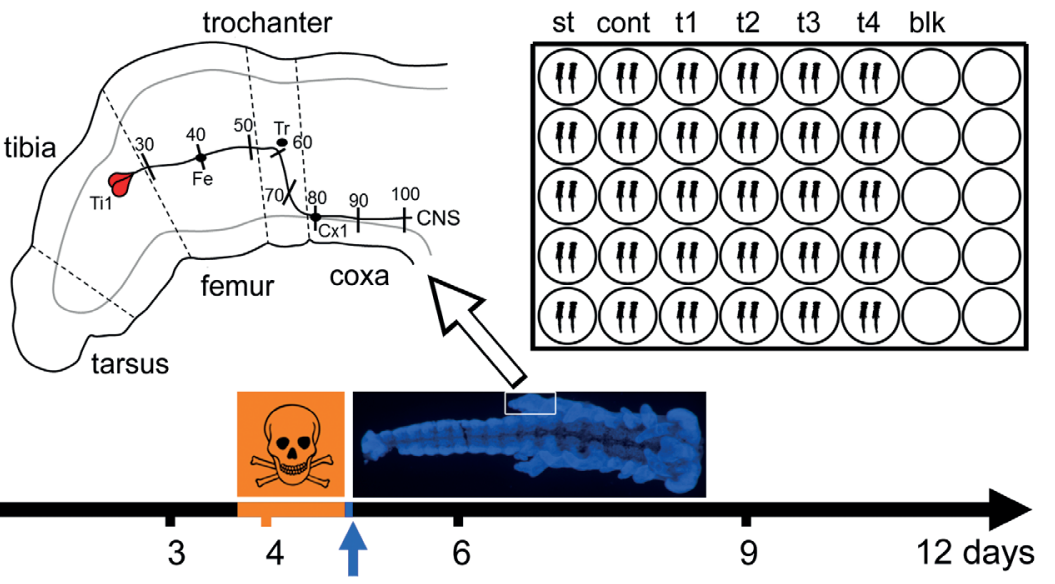

Fig. 1: DNT assay on embryonic locust limb bud pioneer neurons

(A) Schematic of hind limb bud of an embryo developed to 35\% until hatching. Development and axon elongation of the sibling pair of Ti1 pioneer neurons (red) is divided into 11 steps from birth (0) to reaching the CNS (100), encountering three guidepost cells (Fe, Tr, Cx1) on their way. (B) Schematic of the assay setup in part of a 48-well plate, each well receiving two embryos (st, start control, fixed before incubation; cont, media control, without toxicant; $\mathrm{t1}$-t4, different concentrations of test compound; blk, blank, media only, no embryos).

(C) Timing of embryonic development and exposure to toxicants. At $30^{\circ} \mathrm{C}$, locust nymphs hatch 12 days after eggs are laid. By the end of day 3 , embryos are staged to $32.5 \%$ of development by external features (example: fixed embryo after labeling with DAPI) and subjected to test compounds for $24 \mathrm{~h}$ in vitro (orange), followed by 2 hours for the viability assay (blue) before fixation and immunofluorescence labeling.

planarian model revealed a high degree of predictability for effects on human CNS development (Hagstrom et al., 2019), even though a significant number of genes upregulated in the developing human cortex are species-specific when compared to mouse (Zeng et al., 2012), or even chimpanzee (Zhang et al., 2011).

In vivo studies bear the problems of undefined exposure parameters such as actual concentrations in the tissue of interest (due to diffusion barriers, metabolism, etc.). Here, we propose an ex vivo systemic approach that addresses some of the complexity of the in vivo situation in a simple embryonic invertebrate preparation, under controlled environment, with easy access for test compounds.

The formation of a functional brain requires the precisely timed navigation of axons within the complex neuronal tissue environment. As shown by antibody blocking experiments, growth cone navigation can depend on membrane-bound or molecular gradients of diffusible semaphorin cues, first discovered in the locust embryo (Kolodkin et al., 1992; Isbister et al., 1999). These are conserved in vertebrates (Luo et al., 1993) including mammalians, where semaphorins play an important role in brain cortex formation (Polleux et al., 2000). In contrast to the vertebrate peripheral nervous system, insect peripheral neurons develop from small sets of specialized epidermal cells (Bate, 1976). During ear- ly locust embryogenesis, a pair of pioneer neurons differentiates within the distal tip of limb bud epithelia. These pioneer neurons establish the first axonal pathway to the CNS, using semaphorin signals as extracellular guidance cues. Later born sensory neurons arise from the limb bud epithelium and follow this pathway. Here, we expose intact locust embryos to chemicals in culture medium and monitor defects in axonal navigation of limb bud pioneer axons. Using a resazurin assay, we quantify general cytotoxicity to identify possible DNT effects of the chemicals (Fig. 1).

\section{Methods}

A detailed description of the methods can be found in the supplementary file ${ }^{1}$. Locust eggs (Locusta migratoria) were collected in batches from the same egg pod (50-60 siblings of the same age) from our crowded laboratory culture. Embryos were carefully staged to $32.5 \%$ of completed embryogenesis according to Bentley et al. (1979), dissected under semi-sterile conditions in serum-free L15 media with 1\% penicillin/streptomycin (Invitrogen) and kept ex ovo in 48 well plates at $30^{\circ} \mathrm{C}$ for $24 \mathrm{~h}$ in the presence of test chemicals in groups of 10 embryos per concentration (two embryos per well). Each experiment included a group

1 doi:10.14573/altex.1901292s 
of media controls and a group of start controls, the latter of which were fixed immediately after dissection (Fig. 1B). After washing for $5 \mathrm{~min}$ in L15, a resazurin reduction viability assay (Alamar Blue, Invitrogen) was performed for $2 \mathrm{~h}$, followed by fixation in $4 \%$ paraformaldehyde for $45 \mathrm{~min}$. Leg bud pioneer neurons were immunolabeled for a neuronal cell marker (anti-HRP, Dianova, 1:2000 preceded by permeabilization in $0.3 \%$ saponin and blocking in 5\% normal rabbit serum for $45 \mathrm{~min}$ each). Labeled neurons were visualized by a biotinylated rabbit anti-goat antiserum (1:250, Dianova) and streptavidin-CY3 (1:250, Sigma) plus $0.1 \mu \mathrm{g} / \mathrm{ml}$ DAPI as a nuclear marker. Defects in axonal outgrowth and navigation of pioneer axons were detected via conventional fluorescence microscopy using Zeiss equipment, or confocal microscopy using a Leica TCS SP5. For each leg bud, elongation of pioneer axons along their predefined pathway was scored between $0 \%$ and $100 \%$ according to the scheme depicted in Fig. $1 \mathrm{~A}$. Values were normalized to the averages of the matched start and media controls. Pooled averages from three independent experiments were plotted in GraphPad Prism 8.0 as means \pm SEM Concentration-response curves were generated by fitting four parameter sigmoidal functions. IC50 values were determined from the curve, unless values below 50\% were not reached. In these cases, the highest used concentrations were used to determine IC50 instead, according to Krug et al. (2013).

\section{Results and discussion}

Locust embryos dissected out of their egg shell continue to develop in serum-free L 15 cell culture medium. Since pioneer neurons of body appendages also extend their axon under these conditions (Seidel and Bicker, 2000), we explored this embryo culture system as a potential in vitro DNT assay. Axon outgrowth from a pair of hindleg pioneer neurons follows a characteristic, stereotype pathway from the tibia leg segment into the central nervous system (Bentley and Caudy, 1983), both in ovo and ex ovo (Fig. 2). However, development is slowed down to about half the normal speed ex ovo, conveniently allowing us to monitor the process of neurite outgrowth within $24 \mathrm{~h}$ that would only take $12 \mathrm{~h}$ in ovo. When embryos staged to $32.5 \%$ of their development are fixed and immunolabeled, their neurites have just begun to grow out (Fig. 2, start). In embryos cultivated in vitro from that stage on, neurites will just have arrived in the central nervous system by $24 \mathrm{~h}$ (Fig. 2, L15 only), as compared to the much further developed limb bud cultivated without prior dissection for the same time (Fig. 2, in ovo). In vitro culture allows free access of chemicals to the embryo and has been successfully used to perturb pioneer neuron development, e.g., by interfering with semaphorin signaling (Kolodkin et al., 1992; Isbister et al., 1999) or the NO-cGMP pathway (Seidel and Bicker, 2000).

We can monitor development and its impairment by presumptive developmental neurotoxicants by defining specific and unspecific endpoints. First, we quantify the progress of the growing neurites along their stereotype pathways by applying a well-defined elongation score scheme (Fig. 1A) that uses recognizable landmarks like guidepost cells (Bentley and Caudy, 1983) or leg segment boundaries. This score is more reliable and also much more quickly obtained than measuring actual neurite length on photomicrographs, which is often confounded by variable three-dimensional positions or distortions of the limb buds. "Start" values obtained from preparations fixed before the sibling embryos were subjected to in vitro culture and test compound exposure had to be subtracted in order to measure only the progress. Progress can be hindered by both simple growth retardation or by pathfinding errors, such as growth in incorrect directions, or defasciculations of the sibling pioneer axons (examples in Fig. 2, colchicine and rotenone).

As a second endpoint, we define the ability of the embryo to reduce resazurin to resorufin (general viability), in order to distinguish between general cytotoxicity and specific effects on stereotype neurite growth patterns. By normalizing to untreated in vitro control embryos, we could measure specific and unspecific effects over a wide range of concentrations (Fig. 3A-E).

Calcium is a key second messenger for regulating cytoskeletal dynamics during axonal elongation (Kater and Mills, 1991; Kater and Rehder, 1995; Zheng and Poo, 2007). Since filopodial dynamics necessary for growth cone motility and pathfinding critically involve $\mathrm{Ca}^{2+}$-signaling also in locust limb bud pioneer neurons (Lau et al., 1999), blocking of calcium channels should interfere with pioneer axon elongation. The blockers of L-type calcium channels, verapamil and diltiazem, effective also in the insect nervous system (Lohr et al., 2005), both inhibited pioneer axon elongation in a dose dependent manner with an IC50 of 261.1 $\mu \mathrm{M}$ and 518.4 $\mu \mathrm{M}$, respectively (Fig. 3A,D). This is in the same range necessary to block $\mathrm{Ca}^{2+}$ channels in insect neurons and glia cells (Lohr et al., 2005), and only 4-6.5 times larger than, for instance, in sensory fibers in mouse spinal cord in vitro (Martinez-Gomez and Lopez-Garcia, 2007). General viability of the embryos was also affected in a dose-dependent manner by both blockers. However, at the IC50 for elongation, viability was still at $78 \%$ and $86 \%$ of control values, respectively. Thus, these calcium channel blockers displayed endpoint specific developmental neurotoxicity in our pioneer axon elongation test system, as expected from calcium imaging data (Lau et al., 1999).

Likewise, general inhibitors of cytoskeletal dynamics should interfere with axon elongation. The inhibitor of actin polymerization, cytochalasin D, inhibited elongation of pioneer axons with an IC50 of $52.2 \mathrm{nM}$, whereas general viability was not impaired even at the highest tested concentration of $333 \mathrm{nM}$ (Fig. 3B). In cell culture assays, such as LUHMES, cytochalasin D is often not recognized as a specific developmental neurotoxicant (Krug et al., 2013). The microtubule inhibitor, colchicine, impaired pioneer axon elongation with an IC50 of $158.4 \mathrm{nM}$, whereas general viability was not impaired at the highest tested concentration, $5 \mu \mathrm{M}$. At high colchicine concentrations, viability measurements above $100 \%$ most likely derive from increased metabolism during apoptotic cell death induced by colchicine (TUNEL assay data not shown). Interestingly, although pioneer axons pursued highly erratic pathways even at moderate colchicine concentrations (Fig. 2), up to $40 \%$ of the pioneer neurons nevertheless reached their final destination, the CNS, even at $5 \mu \mathrm{M}$ colchicine (Fig. 3E). In cell culture, colchicine has been reported to reduce 


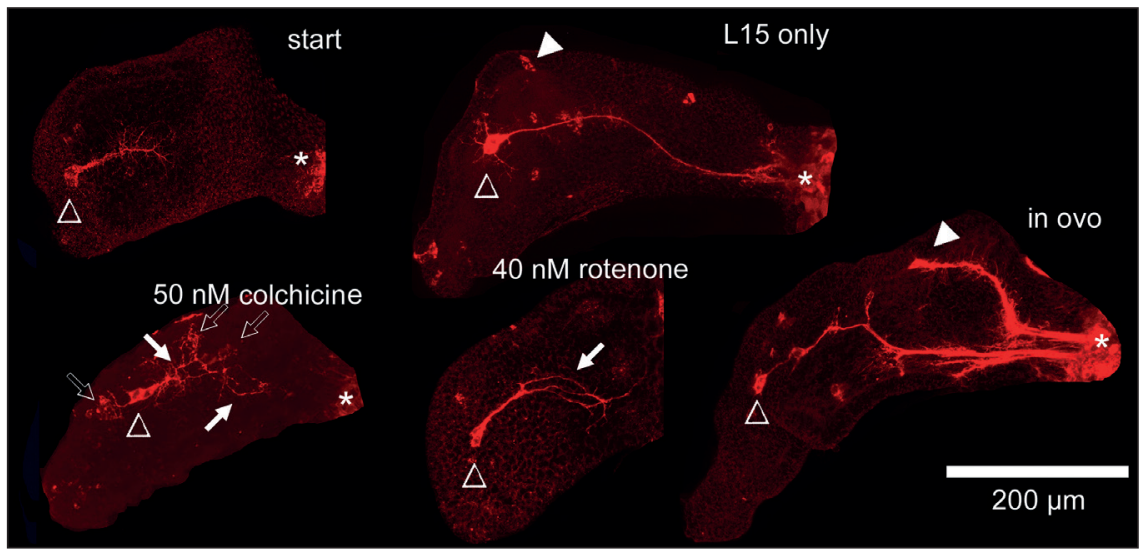

Fig. 2: Embryonic locust limb bud pioneer neurons in vitro

Pioneer neurons immunolabelled for a neuronal cell surface marker (anti-HRP), either fixed immediately without incubation (start) or after $24 \mathrm{~h}$ in culture, either in ovo or ex ovo, in the absence (L15 only) or presence of test compounds $(40 \mathrm{nM}$ rotenone, $50 \mathrm{nM}$ colchicine). Open triangles, pioneer neuron cell bodies; asterisks, CNS; open arrows, growth direction mistakes; filled arrows, defasciculation events; filled triangles, neurons born later during development.

neurite elongation at much lower concentrations (e.g., IC50 of $4 \mathrm{nM}$ for LUHMES cells, Krug et al., 2013), whereas in behavioral assays on whole zebrafish larvae, colchicine had no adverse effect at concentrations up to $30 \mu \mathrm{M}$ (Dach et al., 2019). In intact tissue, axonal growth cones can react via filopodial contacts to a variety of guidance cues such as guidepost cells, compounds of the extracellular matrix, as well as gradients of soluble or cell surface bound factors. All of these cues are absent in an isolated cell culture situation where neurite outgrowth might depend much less on actin driven filopodial movements than on forces generated by microtubule-protein interactions (Roossien et al., 2013; Athamneh et al., 2017). This could explain why actin inhibitors can be far more potent developmental neurotoxicants in tissue than in cell culture, and vice versa for microtubule inhibitors. It also emphasizes the significance of tissue culture-based test systems complementary to cell-based test systems for the translation of in vitro data to prediction of in vivo effects.

The pesticide rotenone, a mitochondrial electron transport chain blocker, is known for its adverse effect on dopaminergic neurons in both mammals and Drosophila (Betarbet et al., 2000; Pamies et al., 2018; Coulom and Birman, 2004), and is thus often used in Parkinson's disease research (Heinz et al., 2017), but it is also a selective inhibitor of axonal outgrowth of human neurons in vitro (Krug et al., 2013). In our experiments, rotenone inhibited pioneer neuron growth with an IC50 of $20.3 \mathrm{nM}$ (Fig. 3C), which is in the same range as found for neurite outgrowth in human neurons (Krug et al., 2013). At this concentration, viability of the embryo (measured by resazurin reduction assay) was less affected (IC50 $56.2 \mathrm{nM}$ ), which identifies rotenone as a specific developmental neurotoxicant also in our insect embryo assay. Rotenone is not only a mitochondrial respiratory chain complex I blocker, but can act in parallel on the cytoskeleton by inhibiting microtubule assembly (Marshall and Himes, 1978; Heinz et al., 2017) and the RhoA/ROCK pathway (Bisbal et al., 2018). Rho kinases (ROCKs) are attractive drug targets for restoring neural outgrowth, since a multitude of the extracellular signals creating a hostile environment for neurite regeneration converge onto the Rho/ROCK pathway, which regulates cytoskeletal dynamics (Mueller et al., 2005). Pharmacological inhibition of the Rho/ ROCK pathway enhances neurite outgrowth in animal models of spinal cord injury and cell cultures of human model neurons (Mueller et al., 2005; Roloff et al., 2015; Krug et al., 2013). In the locust embryo, application of $50 \mu \mathrm{M}$ of the small-molecule ROCK inhibitor, Y27632, significantly alleviated rotenone-induced reduction of pioneer axon elongation (Fig. 3F), confirming a DNT-specific action of rotenone.

Our axon elongation experiments also classified endpoint specific inhibitors of calcium-dependent growth cone motility and general cytoskeletal inhibitors as specific effectors, compared to general cytotoxicity - a key requirement for a DNT assay (Aschner et al., 2017). However, the nervous system contributes only $\sim 10 \%$ of cells to the whole embryo, and other (e.g., proliferating) cells may be more susceptible to toxicants than postmitotic neurons. In our preparations, all neurons with an elongation score greater than zero had non-fragmented neurites and most bore filopodia - which indicates that cells were still alive at the time of fixation. Thus, the biochemical measurements of general cytotoxicity to the embryo should not be overinterpreted. The development of an alternative viability assay, sensitive to single neuron cytotoxicity, would be advantageous.

The advantage of this assay as an alternative to animal experimentation in DNT screening is that our assay is performed on an intact invertebrate embryo cultured in serum-free medium. This assay is focusing on an individually identifiable pair of neurons critically involved in establishing the first neural pathway from the periphery towards the CNS (Bentley and Caudy, 1983; Isbister et al., 1999). Since the shape of these parallel-projecting pioneer neurons remains fairly constant from embryo to embryo, experimentally-induced changes in neuron geometry can be easily resolved. We are currently exploring a 3D imaging method (Lorbeer et al., 2011) to quantify abnormal geometries in the wiring of the nervous system under the influence of DNT compounds. On a phylogenetic scale, the embryo of a migratory locust is clearly remote from any vertebrate embryo, e.g., fish or chicken, that are also used in DNT research. This minimizes, if not obviates any ethical issues about the use of a pest insect embryo as a test organism.

On the other hand, it is striking that insect pioneer and mammalian pyramidal neurons in the cortex rely on members of the conserved semaphorin family as extracellular guidance cues 


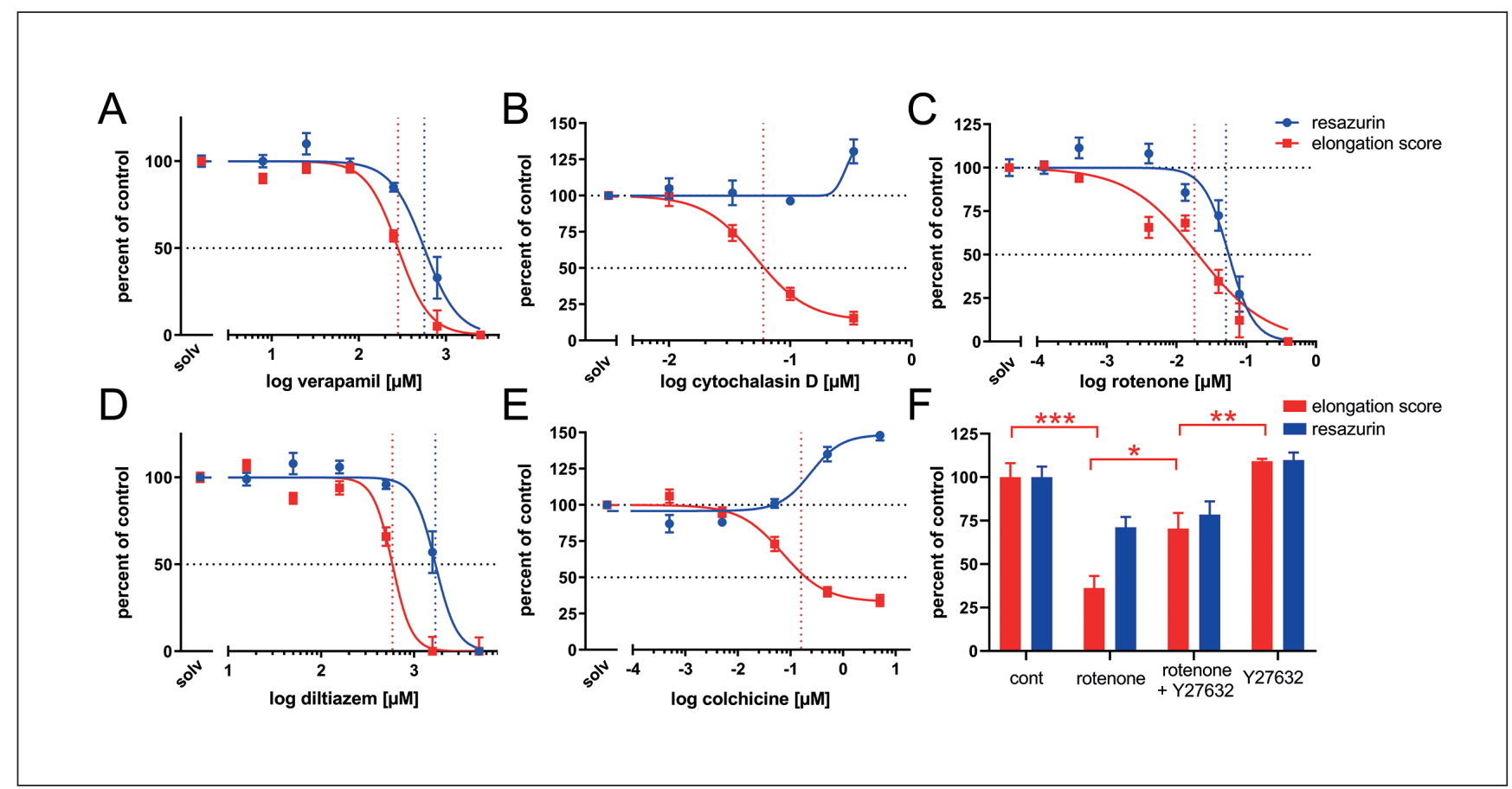

Fig. 3: Concentration-response curves of general cytotoxicity (resazurin, blue), and directed pioneer axon elongation according to the schematic in Fig. 1A (red)

(A-E) Each data point is the average $( \pm$ SEM) of at least 18 measurements normalized to untreated in vitro controls and non-incubated start controls taken from at least three different independent experiments. ( $F$ ) Partial recovery of axon elongation impairment caused by $40 \mathrm{nM}$ rotenone, by co-application of $50 \mu \mathrm{M}$ of the ROCK inhibitor, Y27632. Each bar is the average $( \pm \mathrm{SEM})$ from three independent experiments $\left({ }^{\star} p<0.05,{ }^{* *} p<0.01,{ }^{* * *} p<0.001\right.$, Kruskal-Wallis test followed by Dunn's post-hoc test with correction for multiple comparisons).

(Kolodkin et al., 1992; Isbister et al., 1999; Polleux et al., 2000). Another conserved mechanism appears to be the differential regulation of intracellular cGMP levels in the semaphorin-mediated neurite outgrowth from the cortical and locust pioneer neurons (Polleux et al., 2000; Seidel and Bicker, 2000). Nevertheless, one has to consider species differences, in particular when thinking about potential DNT effects on higher cognitive functions of the human brain, which cannot possibly be addressed in insect embryos. Currently, we are calibrating the assay against a range of positive reference compounds with known DNT potential in humans and negative compounds, which are toxic but have no specific DNT potential (Aschner et al., 2017). This will enable us to develop a prediction model.

Eventually, this insect preparation might serve as a complementary test system to other alternative DNT testing methods of neurite outgrowth and, as part of a test battery (Bal-Price et al., 2018 b), contribute to positive identification of DNT compounds in case the assay performance is satisfying. To date, there are few in vitro tests available that address DNT in intact systems, and the most widely used test on zebrafish involves experiments on postembryonic fish larvae, i.e., vertebrate animals. Another potential application for the pharmaceutical industry lies in the reduction and replacement of animal experiments for the screening of drugs that enhance neurite outgrowth.

\section{References}

Aschner, M., Ceccatelli, S., Daneshian, M. et al. (2017). Reference compounds for alternative test methods to indicate developmental neurotoxicity (DNT) potential of chemicals: Example lists and criteria for their selection and use. ALTEX 34, 4974. doi:10.14573/altex.1604201

Athamneh, A. I. M., He, Y., Lamoureux, P. et al. (2017). Neurite elongation is highly correlated with bulk forward translocation of microtubules. Sci Rep 7, 7292. doi:10.1038/s41598-01707402-6

Avila, D., Helmcke, K. and Aschner, M. (2012). The Caenorhabiditis elegans model as a reliable tool in neurotoxicology. Hum Exp Toxicol 31, 236-243. doi:10.1177/0960327110392084

Bal-Price, A. K., Hogberg, H. T., Crofton, K. M. et al. (2018a). Recommendation on test readiness criteria for new approach methods in toxicology: Exemplified for developmental neurotoxicity. ALTEX 35, 306-352. doi:10.14573/altex.1712081

Bal-Price, A., Pistollato, F., Sachana, M. et al. (2018b). Strategies to improve the regulatory assessment of developmental neurotoxicity (DNT) using in vitro methods. Toxicol Appl Pharmacol 354, 7-18. doi:10.1016/j.taap.2018.02.008

Bate, M. (1976). Pioneer neurons in an insect embryo. Nature 256, 664-665. doi:10.1038/260054a0

Bentley, D., Keshishian, H., Shankland, M. and Toroian-Ray- 
mond, A. (1979). Quantitative staging of embryonic development of the grasshopper, Schistocerca nitens. J Embryol Exp Morph 54, 47-74. http://dev.biologists.org/content/54/1/47.long Bentley, D. and Caudy, M. (1983). Navigational substrates for peripheral pioneer growth cones: Limb-axis polarity cues, limbsegment boundaries, and guidepost neurons. Cold Spring Harb Symp Quant Biol 48 Pt 2, 573-585. doi:10.1101/sqb. 1983.048.01.062

Betarbet, R., Sherer, T. B., MacKenzie, G. et al. (2000). Chronic systemic pesticide exposure reproduces features of Parkinson's disease. Nat Neurosci 3, 1301-1306. doi:10.1038/81834

Bisbal, M., Remedi, M., Quassollo, G. et al. (2018). Rotenone inhibits axonogenesis via an Lfc/RhoA/ROCK pathway in cultured hippocampal neurons. J Neurochem 146, 570-584. doi:10.1111/jnc. 14547

Coecke, S., Goldberg, A. M., Allen, S. et al. (2007). Workgroup report: Incorporating in vitro alternative methods for developmental neurotoxicity into international hazard and risk assessment strategies. Environ Health Perspect 115, 924-931. doi:10.1289/ehp.9427

Coulom, H. and Birman, S. (2004). Chronic exposure to rotenone models sporadic Parkinson's disease in Drosophila melanogaster. $J$ Neurosci 24, 10993-10998. doi:10.1523/JNEUROSCI. 2993-04.2004

Crofton, K. M., Mundy, W. R., Lein, P. J. et al. (2011). Developmental neurotoxicity testing: Recommendations for developing alternative methods for the screening and prioritization of chemicals. ALTEX 28, 9-15. doi:10.14573/altex.2011.1.009

Dach, K., Yaghoobi, B., Schmuck, M. R. et al. (2019). Teratological and behavioral screening of the national toxicology program 91-compound library in zebrafish (Danio rerio). Toxicol Sci 167, 77-91. doi:10.1093/toxsci/kfy266

Delp, J., Gutbier, S., Klima, S. et al. (2018). A high-throughput approach to identify specific neurotoxicants/ developmental toxicants in human neuronal cell function assays. ALTEX 35, 235-253. doi:10.14573/altex.1712182

Frank, C. L., Brown, J. P., Wallace, K. et al. (2018). Defining toxicological tipping points in neuronal network development. Toxicol Appl Pharmacol 354, 81-93. doi:10.1016/j. taap.2018.01.017

Fritsche, E., Alm, H., Baumann, J. et al. (2015). Literature review on in vitro and alternative developmental neurotoxicity (DNT) testing methods. EFSA Supporting Publication 2015, EN-778. doi:10.2903/sp.efsa.2015.EN-778

Fritsche, E., Crofton, K. M., Hernandez, A. F. et al. (2017). OECD/EFSA workshop on developmental neurotoxicity (DNT): The use of non-animal test methods for regulatory purposes. ALTEX 34, 311-315. doi:10.14573/altex.1701171

Fritsche, E., Barenys, M., Klose, J. et al. (2018). Current availability of stem cell-based in vitro methods for developmental neurotoxicity (DNT) testing. Toxicol Sci 165, 21-30. doi:10.1093/ toxsci/kfy 178

Hagstrom, D., Truong, S. Q., Tanguay R. et al. (2019). Comparative analysis of zebrafish and planarian model systems for developmental neutrotoxicity screenings using an 87-compund library. Toxicol Sci 167, 16-25. doi:10.1093/toxsci/kfy180
Heinz, S., Freyberger, A., Lawrenz, B. et al. (2017). Mechanistic investigations of the mitochondrial complex I inhibitor rotenone in the context of pharmacological and safety evaluation. Sci Rep 7, 45465. doi:10.1038/srep45465

Isbister, C. M., Tsai, A., Wong, S. T. et al. (1999). Discrete roles for secreted and transmembrane semaphorins in neuronal growth cone guidance in vivo. Development 126, 2007-2019. http://dev.biologists.org/content/126/9/2007.long

Kater, S. B. and Mills, L. R. (1991). Regulation of growth cone behavior by calcium. J Neurosci 11, 891-899. doi:10.1523/ JNEUROSCI.11-04-00891.1991

Kater, S. B. and Rehder, V. (1995). The sensory-motor role of growth cone filopodia. Curr Opin Neurobiol 5, 68-74. doi:10.1016/0959-4388(95)80089-1

Kolodkin, A. L., Matthes, D. J., O’Connor, T. P. et al. (1992). Fasciclin IV: Sequence, expression, and function during growth cone guidance in the grasshopper embryo. Neuron 9, 831-845. doi:10.1016/0896-6273(92)90237-8

Krug, A. K., Balmer, N. V., Matt, F. et al. (2013). Evaluation of a human neurite growth assay as specific screen for developmental neurotoxicants. Arch Toxicol 87, 2215-2231. doi:10.1007/ s00204-013-1072-y

Lau, P. M., Zucker, R. S. and Bentley, D. (1999). Induction of filopodia by direct local elevation of intracellular calcium ion concentration. J Cell Biol 145, 1265-1275. doi:10.1083/ jcb.145.6.1265

Lein, P., Silbergeld, E., Locke, P. and Goldberg, A. M. (2005). In vitro and other alternative approaches to developmental neurotoxicity testing (DNT). Environ Toxicol Pharmacol 19, 735744. doi:10.1016/j.etap.2004.12.035

Lohr, C., Heil, J. E. and Deitmer, J. W. (2005). Blockage of voltage-gated calcium signaling impairs migration of glial cells in vivo. Glia 50, 198-211. doi:10.1002/glia.20163

Lorbeer, R. A., Heidrich, M., Lorbeer, C. et al. (2011). Highly efficient 3D fluorescence microscopy with a scanning laser optical tomograph. Opt Express 19, 5419-5430. doi:10.1364/ OE.19.005419

Luo, Y., Raible, D. and Raper, J. A. (1993). Collapsin: A protein in brain that induces the collapse and paralysis of neuronal growth cones. Cell 75, 217-227. doi:10.1016/00928674(93)80064-L

Marshall, L. E. and Himes, R. H. (1978). Rotenone inhibition of tubulin self-assembly. Biochim Biophys Acta 543, 590-594. doi:10.1016/0304-4165(78)90315-X

Martinez-Gomez, J. and Lopez-Garcia, J. A. (2007). Simultaneous assessment of the effects of L-type current modulators on sensory and motor pathways of the mouse spinal cord in vitro. Neuropharmacology 53, 464-471. doi:10.1016/j.neuropharm. 2007.06.007

Mueller, B. K., Mack, H. and Teusch, N. (2005). Rho kinase, a promising drug target for neurological disorders. Nat Rev Drug Discov 4, 387-398. doi:10.1038/nrd1719

Pamies, D., Block, K., Lau, P. et al. (2018). Rotenone exerts developmental neurotoxicity in a human brain spheroid model. Toxicol Appl Pharmacol 354, 101-114. doi:10.1016/j.taap. 2018.02.003 
Polleux, F., Morrow, T. and Ghosh, A. (2000). Semaphorin 3A is a chemoattractant for cortical apical dendrites. Nature 404, 567-573. doi: $10.1038 / 35007001$

Rand, M. D. (2010). Drosophotoxicology: The growing potential for Drosophila in neurotoxicology. Neurotoxicol Teratol 32, 74-83. doi:10.1016/j.ntt.2009.06.004

Roloff, F., Scheiblich, H., Dewitz, C. et al. (2015). Enhanced neurite outgrowth of human model (NT2) neurons by small-molecule inhibitors of Rho/ROCK signaling. PLoS One 10, e0118536. doi:10.1371/journal.pone.0118536

Roossien, D. H., Lamoureux, P., Van Vactor, D. and Miller, K. E. (2013). Drosophila growth cones advance by forward translocation of the neuronal cytoskeletal meshwork in vivo. PLoS One 8, e80136. doi:10.1371/journal.pone.0080136

Sánchez-Soriano, N., Tear, G., Whitington, P. and Prokop, A. (2007). Drosophila as a genetic and cellular model for studies on axonal growth. Neural Dev 2, 9. doi:10.1186/1749-8104-2-9

Seidel, C. and Bicker, G. (2000). Nitric oxide and cGMP influence axonogenesis of antennal pioneer neurons. Development 127, 4541-4549. http://dev.biologists.org/content/127/21/4541.long

Stern, M., Gierse, A., Tan, S. and Bicker, G. (2014). Human Ntera2 cells as a predictive in vitro test system for developmental neurotoxicity. Arch Toxicol 88, 127-136. doi:10.1007/ s00204-013-1098-1
Zheng, J. Q. and Poo, M. M. (2007). Calcium signaling in neuronal motility. Annu Rev Cell Dev Biol 23, 375-404. doi:10.1146/ annurev.cellbio.23.090506.123221

Zeng, H., Shen, E. H., Hohmann, J.G. et al. (2012). Large-scale cellular-resolution gene profiling in human neocortex reveals species-specific molecular signatures. Cell 149, 483-496. doi:10.1016/j.cell.2012.02.052

Zhang, Y. E., Landback, P., Vibranovski, M. D. and Long, M. (2011). Accelerated recruitment of new brain development genes into the human genome. PLoS Biol 9, e1001179. doi:10.1371/journal.pbio.1001179

\section{Conflict of interest}

The authors declare they have no conflict of interest.

\section{Acknowledgements}

We acknowledge the funding by the German Federal Ministry of Education and Research (BMBF) project 031L0062A. This publication was supported by Deutsche Forschungsgemeinschaft and University of Veterinary Medicine Hannover, Foundation within the funding program Open Access Publishing. 